\title{
Neutrinoless Double Beta Decay and Lepton Flavor Violation
}

\author{
V. Cirigliano, ${ }^{1}$ A. Kurylov, ${ }^{1}$ M. J. Ramsey-Musolf, ${ }^{1,2,3}$ and P. Vogel ${ }^{1}$ \\ ${ }^{1}$ Kellogg Radiation Laboratory, California Institute of Technology, Pasadena, California 91125, USA \\ ${ }^{2}$ Institute for Nuclear Theory, University of Washington, Seattle, Washington 98195, USA \\ ${ }^{3}$ Department of Physics, University of Connecticut, Storrs, Connecticut 06269, USA
}

(Received 21 June 2004; published 1 December 2004)

\begin{abstract}
We point out that extensions of the standard model with low scale $(\sim \mathrm{TeV})$ lepton number violation (LNV) generally lead to a pattern of lepton flavor violation (LFV) experimentally distinguishable from the one implied by models with grand unified theory scale LNV. As a consequence, muon LFV processes provide a powerful diagnostic tool to determine whether or not the effective neutrino mass can be deduced from the rate of neutrinoless double beta decay. We discuss the role of $\mu \rightarrow e \gamma$ and $\mu \rightarrow e$ conversion in nuclei, which will be studied with high sensitivity in forthcoming experiments.
\end{abstract}

DOI: 10.1103/PhysRevLett.93.231802

In the past few years convincing experimental evidence for neutrino oscillations has been collected [1], implying that neutrinos are massive particles, with masses much smaller than those of other known fermions. Since the standard model of particle physics assumes that neutrinos are massless, the study of neutrino mass and the properties of massive neutrinos provides important clues about a more fundamental theory that goes beyond the standard model. Among the most urgent open questions in neutrino physics are the determination of (i) neutrino charge conjugation properties (Dirac or Majorana) and (ii) the absolute mass scale in the spectrum.

The study of neutrinoless double beta decay $(0 \nu \beta \beta)$ can help address these issues. For one, observation of this $\Delta L=2$ process would establish the existence of total lepton number violation (LNV), thereby implying that neutrinos are massive Majorana particles [2]. Ideally, the observation of $0 \nu \beta \beta$ would also help determine the absolute scale of neutrino mass, since the total decay rate $\left(\Gamma_{0 \nu \beta \beta}\right)$ arising from light Majorana neutrinos is proportional to the square of the effective mass, $m_{\beta \beta}$ (defined precisely below). It has long been recognized, however, that the extraction of $m_{\beta \beta}$ from $\Gamma_{0 \nu \beta \beta}$ is problematic, since LNV interactions involving heavy $(\sim \mathrm{TeV})$ particles can make comparably important contributions to the rate. Thus, in the absence of additional information about the mechanism responsible for $0 \nu \beta \beta$, one could not unambiguously infer $m_{\beta \beta}$ from $\Gamma_{0 \nu \beta \beta}$.

In this Letter, we show that experimental searches for lepton flavor violation (LFV) involving charged leptons can help to address this problem by providing a powerful "diagnostic tool" for establishing the $0 \nu \beta \beta$ mechanism. Here, we focus on the standard model forbidden processes $\mu \rightarrow e \gamma$ and $\mu \rightarrow e$ conversion in nuclei that will be studied with unprecedented sensitivity in the forthcoming MEG [3] and MECO [4] experiments, respectively. The relevant branching ratios are $B_{\mu \rightarrow e \gamma}=\Gamma(\mu \rightarrow$ $e \gamma) / \Gamma_{\mu}^{(0)}$ and $B_{\mu \rightarrow e}=\Gamma_{\text {conv }} / \Gamma_{\text {capt }}$, where $\mu \rightarrow e \gamma$ is normalized to the standard muon decay rate $\Gamma_{\mu}^{(0)}=$
PACS numbers: $14.60 . \mathrm{Pq}, 11.30 . \mathrm{Hv}, 23.40 . \mathrm{Bw}$

$\left(G_{F}^{2} m_{\mu}^{5}\right) /\left(192 \pi^{3}\right)$, while $\mu \rightarrow e$ conversion is normalized to the capture rate $\Gamma_{\text {capt }}$. The new experiments will probe $B_{\mu \rightarrow e \gamma}$ and $B_{\mu \rightarrow e}$ at levels that would be sensitive to the effects of LFV induced by interactions involving TeVscale particles. Since models for the generation of Majorana neutrino masses typically also imply the existence of such interactions, studies of charged lepton LFV can also provide insight into the mechanism of $0 \nu \beta \beta$.

The main discriminating quantity in our analysis is the ratio $\mathcal{R}=B_{\mu \rightarrow e} / B_{\mu \rightarrow e \gamma}$. We find the following: (i) Observation of both the LFV muon processes $\mu \rightarrow e$ and $\mu \rightarrow e \gamma$ with relative ratio $\mathcal{R} \sim 10^{-2}$ implies, under generic conditions, that $\Gamma_{0 \nu \beta \beta} \sim m_{\beta \beta}^{2}$. (ii) On the other hand, the observation of LFV muon processes with relative ratio $\mathcal{R} \gg 10^{-2}$ opens up many possibilities, including nontrivial LNV dynamics at the TeV scale (whose effect on $0 \nu \beta \beta$ has to be analyzed on a case by case basis). Therefore, in this scenario no definite conclusion on $0 \nu \beta \beta$ can be drawn based on LFV rates. (iii) Nonobservation of LFV in muon processes in forthcoming experiments would imply either that the scale of nontrivial LFV and LNV is above a few $\mathrm{TeV}$, and thus $\Gamma_{0 \nu \beta \beta} \sim m_{\beta \beta}^{2}$, or that any TeV-scale LNV is approximately flavor diagonal. (If only one process is observed, the deduced constraint on $\mathcal{R}$ may still be of use for our analysis.) Below, we explain the basis for the above observations and discuss the requirements on scenarios that might circumvent them.

In general, $0 \nu \beta \beta$ can be generated by (i) light Majorana $\nu$ exchange (helicity flip and nonflip) or (ii) heavy particle exchange (see, e.g., [5,6]), resulting from LNV dynamics at some scale above the electroweak one. The relative size of heavy $\left(A_{H}\right)$ versus light particle $\left(A_{L}\right)$ exchange contributions to the decay amplitude can be crudely estimated as follows [7]:

$$
A_{L} \sim G_{F}^{2} \frac{m_{\beta \beta}}{\bar{k}^{2}}, \quad A_{H} \sim G_{F}^{2} \frac{M_{W}^{4}}{\Lambda^{5}}, \quad \frac{A_{H}}{A_{L}} \sim \frac{M_{W}^{4} \bar{k}^{2}}{\Lambda^{5} m_{\beta \beta}},
$$


where $m_{\beta \beta}=\sum_{i} U_{e i}^{2} m_{\nu_{i}}$ is the effective Majorana mass ( $U_{\ell n}$ being the light neutrino mixing matrix), $\bar{k}^{2} \sim$ $(50 \mathrm{MeV})^{2}$ is the typical light neutrino virtuality, and $\Lambda$ is the heavy scale relevant to the LNV dynamics. Therefore, $A_{H} / A_{L} \sim O(1)$ for $m_{\beta \beta} \sim 0.1-0.5 \mathrm{eV}$ and $\Lambda \sim 1 \mathrm{TeV}$, and thus the LNV dynamics at the TeV scale leads to similar $0 \nu \beta \beta$ decay rate as the exchange of light Majorana neutrinos with effective mass $m_{\beta \beta} \sim 0.1-0.5 \mathrm{eV}$.

By itself, the $0 \nu \beta \beta$ lifetime measurement does not provide the means for determining the underlying mechanism. The spin-flip and nonflip exchange can, in principle, be distinguished by the measurement of the singleelectron spectra or polarization (see, e.g., [8]). In most cases, however, the observation of the emitted electrons does not distinguish between light Majorana or heavy particle exchange. Thus, one must look for phenomenological consequences of the different mechanisms other than observables directly associated with $0 \nu \beta \beta$. Here we point out the link between $0 \nu \beta \beta$ mechanisms and muon LFV processes.

It is useful to formulate the problem in terms of effective low energy interactions obtained after integrating out the heavy degrees of freedom that induce LNV and LFV dynamics. Relevant quantities in this context are the LNV and LFV scales, which in general may be distinct. As long as both scales are well above the weak scale, then $\Gamma_{0 \nu \beta \beta} \sim m_{\beta \beta}^{2}$, and one does not expect to observe LFV signals in forthcoming experiments. In scenarios with high scale LNV $\left(\Gamma_{0 \nu \beta \beta} \sim m_{\beta \beta}^{2}\right)$ but possibly low scale LFV, one can have different LFV signatures: in models such as supersymmetric grand unified theories (SUSYGUT) [9] or SUSY seesaw [10], one expects $\mathcal{R} \sim 10^{-2}$, while in models based on $E_{6}$ or showing nondecoupling effects one expects $\mathcal{R} \gg 10^{-2}$ [11,12]. Finally, the case where the scales of LNV and LFV are both relatively low $(\lesssim \mathrm{TeV}$ ) is more subtle, as this scenario might lead to observable LFV and at the same time to ambiguities in interpreting a positive signal in $0 \nu \beta \beta$. We show below that in models with $\mathrm{TeV}$ scale $|\Delta L|=1,2$ interactions the typical LFV signature is $\mathcal{R} \gg 10^{-2}$. The above observations allow one to establish the criteria enumerated at the beginning of this Letter.

We now discuss in detail the case of low LNV/LFV scale. Denoting the new physics scale by $\Lambda$, the effective Lagrangian for $0 \nu \beta \beta$ has the form

$$
\mathcal{L}_{0 \nu \beta \beta}=\sum_{i} \frac{\tilde{c}_{i}}{\Lambda^{5}} \tilde{O}_{i} \quad \tilde{O}_{i}=\bar{q} \Gamma_{1} q \bar{q} \Gamma_{2} q \bar{e} \Gamma_{3} e^{c},
$$

where we have suppressed the flavor and Dirac structures for simplicity (a complete list of the dimension nine operators $\tilde{O}_{i}$ can be found in Ref. [6]). For the LFV interactions, one has

$$
\mathcal{L}_{\mathrm{LFV}}=\sum_{i} \frac{c_{i}}{\Lambda^{2}} O_{i}
$$

and a complete operator basis can be found in Refs. $[13,14]$. The LFV operators relevant to our analysis are of the following type:

$$
\begin{aligned}
O_{\sigma L} & =\frac{e}{(4 \pi)^{2}} \bar{\ell}_{i L} \sigma_{\mu \nu} i \not D \ell_{j L} F^{\mu \nu}+\text { H.c., } \\
O_{\ell L} & =\bar{\ell}_{i L} \ell_{j L}^{c} \bar{\ell}_{k L}^{c} \ell_{m L}, \quad O_{\ell q}=\bar{\ell}_{i} \Gamma_{\ell} \ell_{j} \bar{q} \Gamma_{q} q,
\end{aligned}
$$

along with their corresponding chiral analogs $(L \leftrightarrow R)$. Since operators of the type $O_{\sigma}$ typically arise at the oneloop level, we explicitly display the loop factor $1 /(4 \pi)^{2}$. On the other hand, in a large class of models, operators of the type $O_{\ell}$ or $O_{\ell q}$ may arise from both tree-level exchange of heavy particles as well as loop effects. With the above choices, the leading contributions to the various $c_{i}$ are nominally of the same size, typically the product of two Yukawa-like couplings or gauge couplings (times flavor mixing matrices).

In terms of these operator definitions, the ratio $\mathcal{R}$ can be written schematically as follows (neglecting flavor indices in the effective couplings and the term with $L \leftrightarrow$ $R)$ :

$$
\begin{aligned}
\mathcal{R}= & \frac{\Phi}{48 \pi^{2}} \mid \eta_{1} e^{2} c_{\sigma L}+e^{2}\left(\eta_{2} c_{\ell L}+\eta_{3} c_{\ell q}\right) \log \frac{\Lambda^{2}}{m_{\mu}^{2}} \\
& +\eta_{4}(4 \pi)^{2} c_{\ell q}+\left.\cdots\right|^{2} /\left[e^{2}\left(\left|c_{\sigma L}\right|^{2}+\left|c_{\sigma R}\right|^{2}\right)\right] .
\end{aligned}
$$

Here, $\eta_{1,2,3,4}$ are numerical factors of $O(1)$, while the overall factor $\Phi / 48 \pi^{2}$ arises from phase space and overlap integrals involving electron and muon wave functions in the nuclear field. For light nuclei $\Phi=\left(Z F_{p}^{2}\right) /\left(g_{V}^{2}+\right.$ $\left.3 g_{A}^{2}\right) \sim O(1)$, where $g_{V, A}$ are the vector and axial nucleon form factors at zero momentum transfer, while $F_{p}$ is the nuclear form factor at $q^{2}=-m_{\mu}^{2}$ [14]. The dots indicate subleading terms, not relevant for our discussion, such as loop-induced contributions to $c_{\ell}$ and $c_{\ell q}$ that are analytic in external masses and momenta. In contrast the logarithmically enhanced loop contribution given by the second term in the numerator of $\mathcal{R}$ plays an essential role. This term arises whenever the operators $O_{\ell L, R}$ and $O_{\ell q}$ appear at tree level in the effective theory and generate one-loop renormalization of $O_{\ell q}$ [13] (see Fig. 1).

The ingredients in Eq. (5) lead to several observations: (i) In the absence of tree level $c_{\ell L}$ and $c_{\ell q}$, one obtains

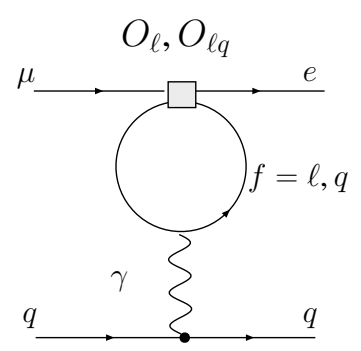

FIG. 1. Loop contributions to $\mu \rightarrow e$ conversion through insertion of operators $O_{\ell}$ or $O_{\ell q}$, generating the large logarithm. 
$\mathcal{R} \sim\left(\Phi \eta_{1}^{2} \alpha\right) /(12 \pi) \sim 10^{-3}-10^{-2}$, due to gauge coupling and phase space suppression. (ii) When present, the logarithmically enhanced contributions compensate for the gauge coupling and phase space suppression, leading to $\mathcal{R} \sim O(1)$. (iii) If present, the tree-level coupling $c_{\ell q}$ dominates the $\mu \rightarrow e$ rate leading to $\mathcal{R} \gg 1$.

Our main claim is that in models with $\mathrm{TeV}$-scale $|\Delta L|=1,2$ interactions and generic flavor content of the couplings one finds $\tilde{c}_{i} / g^{2} \sim c_{\ell L}, c_{\ell R}, c_{\ell q}$ ( $g$ is a gauge coupling), and thus a short-distance contribution to $0 \nu \beta \beta$ is accompanied by $\mathcal{R} \gg 10^{-2}$. We now illustrate this statement in two explicit cases: the minimal supersymmetric standard model (MSSM) with $R$-parity violation (RPV-SUSY) and the left-right symmetric model (LRSM).

$R P V$-SUSY.-If one does not impose $R$-parity conservation $\left[R=(-1)^{3(B-L)+2 s}\right]$, the MSSM superpotential includes, in addition to the standard Yukawa terms, lepton and baryon number violating interactions, compactly written as (see, e.g., [15])

$$
\begin{aligned}
W_{\mathrm{RPV}}= & \lambda_{i j k} L_{i} L_{j} E_{k}^{c}+\lambda_{i j k}^{\prime} L_{i} Q_{j} D_{k}^{c}+\lambda_{i j k}^{\prime \prime} U_{i}^{c} D_{j}^{c} D_{k}^{c} \\
& +\mu_{i}^{\prime} L_{i} H_{u},
\end{aligned}
$$

where $L$ and $Q$ represent lepton and quark doublet superfields, while $E^{c}, U^{c}$, and $D^{c}$ are lepton and quark singlet superfields. The simultaneous presence of $\lambda^{\prime}$ and $\lambda^{\prime \prime}$ couplings would lead to an unacceptably large proton decay rate (for SUSY mass scale $\Lambda_{\text {SUSY }} \sim \mathrm{TeV}$ ), so we focus on the case of $\lambda^{\prime \prime}=0$ and set $\mu^{\prime}=0$ without loss of generality [15]. In this case, the lepton number is violated by the remaining terms in $W_{\mathrm{RPV}}$, leading to short-distance contributions to $0 \nu \beta \beta$, with typical coefficients [cf. Eq. (2)]

$$
\frac{\tilde{c}_{i}}{\Lambda^{5}} \sim \frac{\pi \alpha_{s}}{m_{\tilde{g}}} \frac{\lambda_{111}^{\prime 2}}{m_{\tilde{f}}^{4}} ; \quad \frac{\pi \alpha_{2}}{m_{\chi}} \frac{\lambda_{111}^{\prime 2}}{m_{\tilde{f}}^{4}},
$$

where $\alpha_{s}, \alpha_{2}$ represent the strong and weak gauge coupling constants, respectively. The RPV interactions also lead to lepton number conserving but lepton flavor violating operators [e.g., Fig. 2(b)], with coefficients [cf. Eq. (3)]

$$
\begin{gathered}
\frac{c_{\ell}}{\Lambda^{2}} \sim \frac{\lambda_{i 11} \lambda_{i 21}^{*}}{m_{\tilde{\nu}_{i}}^{2}}, \frac{\lambda_{i 11}^{*} \lambda_{i 12}}{m_{\tilde{\nu}_{i}}^{2}}, \quad \frac{c_{\ell q}}{\Lambda^{2}} \sim \frac{\lambda_{11 i}^{\prime *} \lambda_{21 i}^{\prime}}{m_{\tilde{d}_{i}}^{2}}, \frac{\lambda_{1 i 1}^{\prime *} \lambda_{2 i 1}^{\prime}}{m_{\tilde{u}_{i}}^{2}}, \\
\frac{c_{\sigma}}{\Lambda^{2}} \sim \frac{\lambda \lambda^{*}}{m_{\tilde{\ell}}^{2}}, \frac{\lambda^{\prime} \lambda^{\prime *}}{m_{\tilde{q}}^{2}},
\end{gathered}
$$

where the flavor combinations contributing to $c_{\sigma}$ can be found in Ref. [16]. Hence, for generic flavor structure of the couplings $\lambda$ and $\lambda^{\prime}$ the underlying LNV dynamics generate both short-distance contributions to $0 \nu \beta \beta$ and LFV contributions that lead to $\mathcal{R} \gg 10^{-2}$.

Existing limits on rare processes strongly constrain combinations of RPV couplings, assuming $\Lambda_{\text {SUSY }}$ is between a few hundred $\mathrm{GeV}$ and $\sim 1 \mathrm{TeV}$. Nonobservation of LFV at future experiments MEG and MECO could be

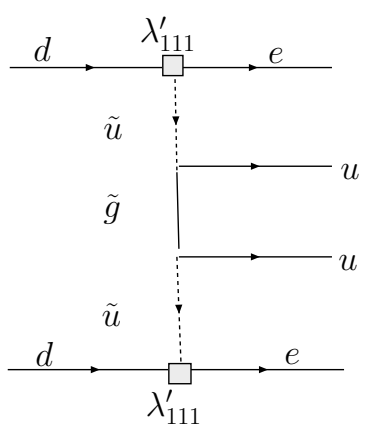

(a)

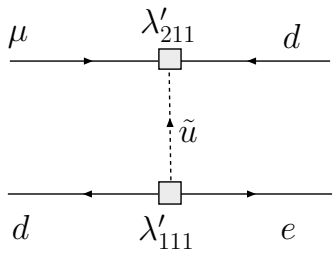

(b)
FIG. 2. Gluino exchange contribution to $0 \nu \beta \beta$ (a), and typical tree-level contribution to $O_{\ell q}$ (b) in RPV-SUSY.

attributed either to a larger $\Lambda_{\text {SUSY }}(>$ a few TeV) or to suppression of couplings that involve mixing among first and second generations. In the former scenario, the shortdistance contribution to $0 \nu \beta \beta$ does not compete with the long distance one [see Eq. (1)], so that $\Gamma_{0 \nu \beta \beta} \sim m_{\beta \beta}^{2}$. On the other hand, if the $\lambda$ and $\lambda^{\prime}$ matrices are nearly flavor diagonal, the exchange of superpartners may still make non-negligible contributions to $0 \nu \beta \beta$.

LRSM.-The LRSM provides a natural scenario for introducing nonsterile, right-handed neutrinos and Majorana masses [17]. The corresponding electroweak gauge group $\mathrm{SU}(2)_{L} \times \mathrm{SU}(2)_{R} \times \mathrm{U}(1)_{B-L}$ breaks down to $\mathrm{SU}(2)_{L} \times \mathrm{U}(1)_{Y}$ at the scale $\Lambda \gtrsim \mathcal{O}(\mathrm{TeV})$. The symmetry breaking is implemented through an extended Higgs sector, containing a bidoublet $\Phi$ and two triplets $\Delta_{L, R}$, whose leptonic couplings generate both Majorana neutrino masses and LFV involving charged leptons:

$$
\begin{aligned}
\mathcal{L}_{Y}^{\text {lept }}= & -\bar{L}_{L}^{i}\left(y_{D}^{i j} \Phi+\tilde{y}_{D}^{i j} \tilde{\Phi}\right) L_{R}^{j}-\overline{\left(L_{L}\right)^{c}} y_{M}^{i j} \tilde{\Delta}_{L} L_{L}^{j} \\
& -\overline{\left(L_{R}\right)^{c}} y_{M}^{i j} \tilde{\Delta}_{R} L_{R}^{j} .
\end{aligned}
$$

Here $\tilde{\Phi}=\sigma_{2} \Phi^{*} \sigma_{2}, \tilde{\Delta}_{L, R}=i \sigma_{2} \Delta_{L, R}$, and leptons belong to two isospin doublets $L_{L, R}^{i}=\left(\nu_{L, R}^{i}, \ell_{L, R}^{i}\right)$. The gauge symmetry is broken through the vacuum expectation values (VEVs) $\left\langle\Delta_{R}^{0}\right\rangle=v_{R},\left\langle\Delta_{L}^{0}\right\rangle=0,\langle\Phi\rangle=\operatorname{diag}\left(\kappa_{1}, \kappa_{2}\right)$. After diagonalization of the lepton mass matrices, LFV arises from both nondiagonal gauge interactions and the Higgs Yukawa couplings. In particular, the $\Delta_{L, R}$-lepton interactions are not suppressed by lepton masses and have the structure $\mathcal{L} \sim \Delta_{L, R}^{++} \bar{\ell}_{i}^{c} h_{i j}\left(1 \pm \gamma_{5}\right) \ell_{j}+$ H.c. The couplings $h_{i j}$ are in general nondiagonal and related to the heavy neutrino mixing matrix [18].

Short-distance contributions to $0 \nu \beta \beta$ arise from the exchange of both heavy $\nu$ s and $\Delta_{L, R}$ (Fig. 3), with

$$
\frac{\tilde{c}_{i}}{\Lambda^{5}} \sim \frac{g_{2}^{4}}{M_{W_{R}}^{4}} \frac{1}{M_{\nu_{R}}} ; \quad \frac{g_{2}^{3}}{M_{W_{R}}^{3}} \frac{h_{e e}}{M_{\Delta}^{2}},
$$

where $g_{2}$ is the weak gauge coupling. LFV operators are also generated through nondiagonal gauge and Higgs vertices, with [18] 


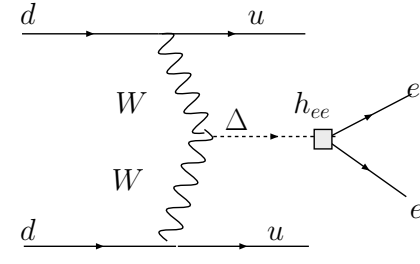

(a)

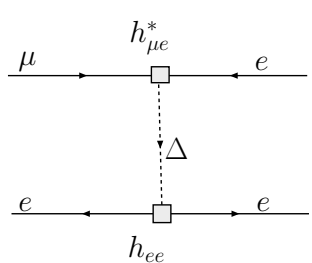

(b)
FIG. 3. Typical doubly charged Higgs contribution to $0 \nu \beta \beta$ (a) and to $O_{\ell}$ (b) in the LRSM.

$$
\frac{c_{\ell}}{\Lambda^{2}} \sim \frac{h_{\mu i} h_{i e}^{*}}{m_{\Delta}^{2}} \quad \frac{c_{\sigma}}{\Lambda^{2}} \sim \frac{\left(h^{\dagger} h\right)_{e \mu}}{M_{W_{R}}^{2}} \quad i=e, \mu, \tau .
$$

Note that the Yukawa interactions needed for the Majorana neutrino mass necessarily imply the presence of LNV and LFV couplings $h_{i j}$ and the corresponding LFV operator coefficients $c_{\ell}$, leading to $\mathcal{R} \sim O(1)$. Again, nonobservation of LFV in the next generation of experiments would typically push $\Lambda$ into the multi-TeV range, thus implying a negligible short-distance contribution to $0 \nu \beta \beta$. As with RPV-SUSY, this conclusion can be evaded by assuming a specific flavor structure, namely, $y_{M}$ approximately diagonal or a nearly degenerate heavy neutrino spectrum.

In both of the foregoing phenomenologically viable models incorporating LNV and LFV at low scale ( $\mathrm{TeV}$ ), one finds $\mathcal{R} \gg 10^{-2}[13,16,18]$. We stress that the basic mechanism at work in these illustrative cases is likely to be generic: low scale LNV interactions $(\Delta L=$ \pm 1 and/or $\Delta L= \pm 2$ ), which in general contribute to $0 \nu \beta \beta$, also generate sizable contributions to $\mu \rightarrow e$ conversion, thus enhancing this process over $\mu \rightarrow e \gamma$. Barring accidental cancellations or explicit fine-tuning of flavor structures, this enhancement happens essentially through tree-level generation of four-fermion operators of the type $O_{\ell q}$ or $O_{\ell}$.

It is possible, of course, that any short-distance LNV sufficiently strong to affect the interpretation of $\Gamma_{0 \nu \beta \beta}$ is also (approximately) flavor diagonal. In this case, one would expect both branching ratios $B_{\mu \rightarrow e \gamma}$ and $B_{\mu \rightarrow e}$ to be below the reach of upcoming LFV searches, and one would need additional phenomenological handles to sort out the mechanism for a nonzero signal in $0 \nu \beta \beta$. Although seemingly unnatural, such a situation is not precluded theoretically. Indeed, analogous flavor finetuning (the phenomenological absence of flavor changing neutral currents) in lepton number conserving modelssuch as the soft sector of the MSSM - is not unheard of.

In conclusion, we have argued that the ratio $\mathcal{R}=$ $B_{\mu \rightarrow e} / B_{\mu \rightarrow e \gamma}$ of muon LFV processes will provide important insight about the mechanism of neutrinoless double beta decay and the use of this process to determine the absolute scale of neutrino mass. Assuming observation of LFV processes in forthcoming experiments, if $\mathcal{R} \sim 10^{-2}$ the mechanism of $0 \nu \beta \beta$ is light Majorana neutrino exchange; if $\mathcal{R} \gg 10^{-2}$, there might be TeVscale LNV dynamics, and no definite conclusion on the mechanism of $0 \nu \beta \beta$ can be drawn based only on LFV processes.

This work was supported in part by the Institute for Nuclear Theory, U.S. DOE Contracts No. DE-FG0388ER40397 and No. DE-FG02-00ER41132, and by NSF Grant No. PHY-0071856.

[1] S. Fukuda et al., Phys. Rev. Lett. 81, 1562 (1998); 82, 2644 (1999); 85, 3999 (2000); Q. R. Ahmad et al., Phys. Rev. Lett. 87, 071301 (2001); 89, 011301 (2002); 89, 011302 (2002); K. Eguchi et al., Phys. Rev. Lett. 90, 021802 (2003).

[2] J. Schechter and J. Valle, Phys. Rev. D 25, 2951 (1982).

[3] G. Signorelli, J. Phys. G 29, 2027 (2003).

[4] J. L. Popp, Nucl. Instrum. Methods Phys. Res., Sect. A 472, 354 (2000).

[5] R. N. Mohapatra, Phys. Rev. D 34, 3457 (1986); J. D. Vergados, Phys. Lett. B 184, 55 (1987); M. Hirsch et al., Phys. Rev. D 53, 1329 (1996); M. Hirsch et al., Phys. Lett. B 374, 7 (1996); A. Fässler et al., Phys. Rev. Lett. 78, 183 (1997); H. Päs et al., Phys. Lett. B 498, 35 (2001); F. Šimkovic and A. Fässler, Prog. Part. Nucl. Phys. 48, 201 (2002).

[6] G. Prezeau, M. Ramsey-Musolf, and P. Vogel, Phys. Rev. D 68, 034016 (2003).

[7] R. N. Mohapatra, Nucl. Phys. B, Proc. Suppl. 77, 376 (1999); for a more refined treatment, see G. Prezeau, hep-ph/0409235.

[8] M. Doi, T. Kotani, and E. Takasugi, Prog. Theor. Phys. Suppl. 83, 1 (1985).

[9] R. Barbieri, L. J. Hall, and A. Strumia, Nucl. Phys. B445, 219 (1995).

[10] F. Borzumati and A. Masiero, Phys. Rev. Lett. 57, 961 (1986).

[11] J. Bernabeu et al., Nucl. Phys. B409, 69 (1993).

[12] D. Tommasini et al., Nucl. Phys. B444, 451 (1995).

[13] M. Raidal and A. Santamaria, Phys. Lett. B 421, 250 (1998).

[14] R. Kitano, M. Koike, and Y. Okada, Phys. Rev. D 66, 096002 (2002).

[15] H. K. Dreiner, in Perspectives on Supersymmetry, edited by G. L. Kane (World Scientific, Singapore, 1998), pp. 462-479.

[16] A. de Gouvea, S. Lola, and K. Tobe, Phys. Rev. D 63, 035004 (2001).

[17] R. N. Mohapatra and G. Senjanovic, Phys. Rev. Lett. 44, 912 (1980); Phys. Rev. D 23, 165 (1981).

[18] V. Cirigliano et al., Phys. Rev. D 70, 075007 (2004). 